\title{
Cholecystitis, CTCAE 5.0
}

National Cancer Institute

\section{Source}

National Cancer Institute. Cholecystitis, CT CAE 5.0. NCI Thesaurus. Code C146788.

A disorder characterized by inflammation involving the gallbladder. It may be associated with the presence of gallstones. 